\title{
The effect of smoothing functions on data obtained from a FFT
}

\author{
MICHAEL YOST, FREDERICK J. BREMNER, R. J. HELMER, \\ and MARIE-ELENA C. CHINO \\ Trinity University, San Antonio, Texas
}

\begin{abstract}
This research investigated the effect on power spectra when data-smoothing functions were used on EEG data prior to submitting them to a FFT. We used two smoothing function options: not using any smoothing function and using the Parzen smoothing function. We developed a program to evaluate each of these functions with real and standard data. When a set of data is submitted to smoothing prior to being submitted to a FFT, there are statistically significant differences in the power spectra obtained from the FFT. This finding holds true for standard waveforms as well as for real EEG data.
\end{abstract}

Several years ago, it became apparent that there was an inordinant amount of variance in the EEG spectra data that we were gathering and analyzing (Bremner, Yost, \& McKenzie, 1982). We decided that there were three potential sources of this variance. The variance could be (1) the natural variability in the brain physiology reaction of subjects, (2) a function of real differences between the brain physiology reactions of subjects performing different tasks, or (3) a result of the way in which we gathered and analyzed our data. We approached the first two potential problems by developing a rigorous singlesubject design and analysis technique (Bremner, Yost, \& Pike, 1984; Eddy \& Moise, 1985; Perez \& Brown, 1984; Yost \& Bremner, 1985). Using the single-subject design and statistical approach, we were able to reduce much of the variance in our EEG studies. However, there was still variance in the data that did not represent the natural brain physiology variability or experimental manipulation. The goal of this study was to determine whether the variances in our data were, in any way, a result of data gathering and analysis techniques and, furthermore, to determine whether there was a way of reducing this variability. More specifically, the objectives of this study were to investigate the effect on EEG spectra of: (1) applying a smoothing function to EEG data prior to the time it is submitted to a fast Fourier transform (FFT); (2) choosing samples of EEG data with different initial phase angles; and (3) using single integer units for spectral windows when the data being gathered may or may not have a dominant frequency that is an integer value.

\section{METHODOLOGY}

In an earlier paper (Yost, Cooper, \& Bremner, 1983), we described the function of a FFT. With this in mind, we began this study by deciding that we would not use

The authors' mailing address is: Department of Psychology, Trinity University, San Antonio, TX 78284. the computer hardware or programs that we usually use in conducting our research. This was a conscious effort to avoid using the scientific tools that we traditionally use that might inadvertently, and unknowingly to us, introduce variance. We also decided to use data generated by the computer with known and proven mathematical equations so that any variance would be predictable and easily explained.

Using an IBM PC-XT, we developed a computer program in BASIC that permitted us to both generate and analyze data. The program generated periodic waves of different forms (sine, saw tooth, isosceles triangle, and square waves) at any chosen frequency or combination of up to three frequencies and at any initial phase angle. Also, the program can, when requested, apply a Hanning or a Parzen smoothing function to any of the waves that it generates. Finally, the program applied a FFT to the data that were generated and produced both a power spectrum and percent power spectrum. The spectra were in $1-\mathrm{Hz}$ bands beginning at $1 \mathrm{~Hz}$ and continuing through $24 \mathrm{~Hz}$. The program, called "Shadow," produced graphs of the initial wave, the smoothed or unsmoothed wave, and the percent power spectrum. Before using the Shadow program, we thoroughly tested and found it to be reliable and valid.

\section{RESULTS}

The study began by generating a series of $124-\mathrm{Hz}$ sine waves. The first wave had a phase angle of $0^{\circ}$, the second a phase angle of $30^{\circ}$, and the third a phase angle of $60^{\circ}$. The remainder were incremented at $30^{\circ}$ with the last at $330^{\circ}$. Two sets of $124-\mathrm{Hz}$ sine waves were generated. The first set of waves was not mathematically smoothed prior to being submitted to a FFT, and the second set was mathematically smoothed using a Parzen (Jenkins \& Watts, 1969) smoothing function prior to being submitted to the FFT. The summary statistics for the spectra of the two sets of waves are contained in Table 1. 
Table 1

Percent Power Distribution of a 4-Hz Wave

\begin{tabular}{|c|c|c|c|c|}
\hline \multirow{2}{*}{$\begin{array}{c}\text { Frequency } \\
\text { Band }\end{array}$} & \multirow[b]{2}{*}{ Mean } & \multirow[b]{2}{*}{ SD } & \multicolumn{2}{|c|}{ Range } \\
\hline & & & High & Low \\
\hline \multicolumn{5}{|c|}{ Not Smoothed } \\
\hline 3 & 0.0 & 0.0 & 0.0 & 0.0 \\
\hline 4 & 100.0 & 0.0 & 100.0 & 100.0 \\
\hline 5 & 0.0 & 0.0 & 0.0 & 0.0 \\
\hline Total Power & 4096.0 & 0.0 & 4096.0 & 4096.0 \\
\hline \multicolumn{5}{|c|}{ Smoothed } \\
\hline 3 & 12.5 & 0.2 & 12.9 & 12.1 \\
\hline 4 & 75.0 & 0.2 & 75.8 & 74.2 \\
\hline 5 & 12.5 & 0.2 & 12.9 & 12.1 \\
\hline Total Power & 1367.0 & 5.0 & 1380.0 & 1362.0 \\
\hline
\end{tabular}

The percent power spectrum of the nonsmoothed wave was exactly as we expected. When a wave having an integer frequency is submitted to a FFT which was designed to measure power in integer frequency bands, all of the power in the wave is accounted for at one frequency. Note that there was no deviation in the $4-\mathrm{Hz}$ percent power across the sample of 12 waves having different initial phase angles. We expected that smoothing a wave prior to submitting it to a FFT would cause some of the power at the dominant frequency to be displaced into the adjacent frequency bands. We did not expect that as much as $25 \%$ of the power at the dominant frequency would be displaced in this manner. The displaced power was equally distributed between the frequency bands on either side of the dominant frequency.

To investigate the percent power displaced into adjacent frequency bands, we generated two series of waves between $4 \mathrm{~Hz}$ and $5 \mathrm{~Hz}$ at $.1-\mathrm{Hz}$ intervals. All of the waves had a $0^{\circ}$ initial phase angle. One series of waves was submitted to a Parzen smoothing function prior to being submitted to a FFT, and the other series was not. The percent power of the unsmoothed waves at each frequency band was used as the baseline against which to compare the percent power displacement of the corresponding smoothed waves. Figure 1 is a graph of the percent power displacement. The solid line indicates the percent reduction in the dominant frequency as a function of the percent of total power found in the dominant frequency. The dotted line indicates the percent of total power which is displaced into each adjacent frequency as the dominant frequency increases in magnitude. When $100 \%$ of the power is in one frequency (which one would not expect to find very often in the real world), $25 \%$ of the power is displaced into the two adjacent frequencies (12.5\% into the left one and $12.5 \%$ into the right one).

The second analysis was conducted in the same manner as the first. The only exception was that in the second analysis, a $4.5-\mathrm{Hz}$ wave was used instead of a 4.0$\mathrm{Hz}$ wave. The summary statistics for the spectra of the two sets of waves are contained in Table 2 . The percent power spectra of the unsmoothed waves were unexpected. The percent power at each $1-\mathrm{Hz}$ frequency band was not the same for waves having different initial phase angles. The ranges and standard deviations of the percent power at each frequency indicate the amount of variation in the percent power as the initial phase angles vary from $0^{\circ}$ to $330^{\circ}$. Note that the variance present in these data was not a function of subjects or laboratory instrumentation, but was, instead, a function of the way in which the data were sampled. Even though the wave that was input to generate these data was $4.5 \mathrm{~Hz}$, only $81.5 \%$ of the total power extracted was in the $4-$ and $5-\mathrm{Hz}$ frequency bands. This means that more than $18 \%$ of the total power was displaced into frequencies other than ones in which we would have logically expected to find them.

The spectra of the smoothed $4.5-\mathrm{Hz}$ data were different from that of the nonsmoothed spectra. Although there were some small variances in the percent power in each of the frequency bands of the smoothed spectra, the percent power at each frequency was very stable. Also, $98.5 \%$ of the total power was accounted for in the 4- and $5-\mathrm{Hz}$ frequency bands, where it would logically be expected, and there was very little displacement of power into adjacent frequencies as we saw in the previous analysis. When the analyses of the smoothed and unsmoothed data are compared, it appears that the smoothed data contain more stable and representative information than do the unsmoothed data.

The third analysis was conducted using the same methodology used in the first analysis. The only exception was that a composite of 4-, 7-, and $10-\mathrm{Hz}$ waves was used as data in the third analysis. The waves were generated with a common initial phase angle, and the phase angle of the composite wave was used to generate the $30^{\circ}$ increments used in the study. The summary statistics for this analysis are contained in Table 3 . The percent power spectrum of the unsmoothed composite wave was as expected. Approximately $33.3 \%$ of the power was found in each of the 4-, 7-, and $10-\mathrm{Hz}$ frequency bands. There was no variation in this percent power distribution, and no power was displaced into the adjacent frequencies.

The percent power spectra of the smoothed composite waves were very different from those of the unsmoothed waves. Approximately $23 \%$ of the power was found in each of the 4-, 7-, and $10-\mathrm{Hz}$ frequency bands, and ap-

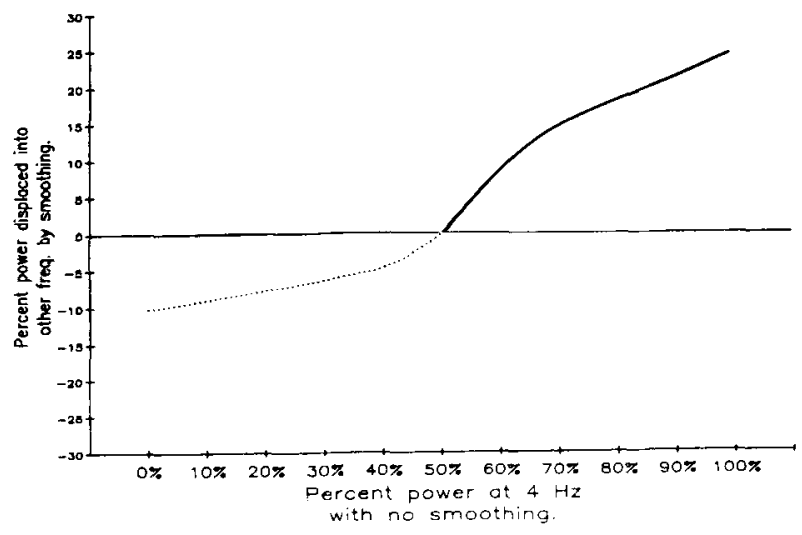

Figure 1. Percent Power Displacement. 
Table 2

Percent Power Distribution of a $4.5-\mathrm{Hz}$ Wave

\begin{tabular}{crrrr}
\hline \multirow{5}{*}{$\begin{array}{c}\text { Frequency } \\
\text { Band }\end{array}$} & Mean & SD & \multicolumn{2}{c}{ Range } \\
\cline { 3 - 5 } & \multicolumn{3}{c}{ Not Smoothed } & Low \\
\hline & 1.9 & 0.90 & 3.1 & 0.6 \\
3 & 3.5 & 1.40 & 6.4 & 2.9 \\
4 & 41.1 & 3.30 & 44.9 & 36.5 \\
5 & 40.4 & 3.60 & 45.5 & 36.1 \\
6 & 4.6 & 0.90 & 5.9 & 3.3 \\
7 & 1.7 & 0.50 & 2.4 & 1.0 \\
Total Power & 4086.0 & 21.00 & 4135.0 & 4036.0 \\
& & Smoothed & & \\
2 & 0.1 & 0.02 & 0.1 & 0.1 \\
3 & 0.6 & 0.04 & 0.6 & 0.5 \\
4 & 49.2 & 0.03 & 49.3 & 49.2 \\
5 & 49.3 & 0.07 & 49.4 & 49.2 \\
6 & 0.6 & 0.04 & 0.6 & 0.5 \\
7 & 0.1 & 0.02 & 0.1 & 0.1 \\
Total Power & 1365.0 & 3.50 & 1373.0 & 1357.0 \\
\hline
\end{tabular}

Table 3

Percent Power Distribution of a Composite Wave $(4,7,10 \mathrm{~Hz})$

\begin{tabular}{|c|c|c|c|c|}
\hline \multirow{2}{*}{$\begin{array}{c}\text { Frequency } \\
\text { Band }\end{array}$} & \multirow[b]{2}{*}{ Mean } & \multirow[b]{2}{*}{$\mathrm{SD}$} & \multicolumn{2}{|c|}{ Range } \\
\hline & & & High & Low \\
\hline \multicolumn{5}{|c|}{ Not Smoothed } \\
\hline 3 & 0.0 & 0.00 & 0.0 & 0.0 \\
\hline 4 & 33.3 & 0.00 & 33.3 & 33.3 \\
\hline 5 & 0.0 & 0.00 & 0.0 & 0.0 \\
\hline 6 & 0.0 & 0.00 & 0.0 & 0.0 \\
\hline 7 & 33.3 & 0.00 & 33.3 & 33.3 \\
\hline 8 & 0.0 & 0.00 & 0.0 & 0.0 \\
\hline 9 & 0.0 & 0.00 & 0.0 & 0.0 \\
\hline 10 & 33.3 & 0.00 & 33.3 & 33.3 \\
\hline 11 & 0.0 & 0.00 & 0.0 & 0.0 \\
\hline 12 & 0.0 & 0.00 & 0.0 & 0.0 \\
\hline Total Power & 12288.0 & 0.00 & 12288.0 & 12288.0 \\
\hline \multicolumn{5}{|c|}{ Smoothed } \\
\hline 3 & 4.6 & 0.10 & 4.8 & 4.4 \\
\hline 4 & 24.6 & 0.15 & 24.9 & 24.4 \\
\hline 5 & 4.6 & 0.10 & 4.9 & 4.4 \\
\hline 6 & 4.4 & 0.00 & 4.4 & 4.4 \\
\hline 7 & 22.4 & 0.10 & 22.7 & 22.2 \\
\hline 8 & 4.4 & 0.00 & 4.4 & 4.4 \\
\hline 9 & 4.6 & 0.10 & 4.9 & 4.4 \\
\hline 10 & 24.6 & 0.15 & 24.9 & 24.4 \\
\hline 11 & 4.6 & 0.10 & 4.8 & 4.4 \\
\hline 12 & 1.1 & 0.02 & 1.2 & 0.9 \\
\hline Total Power & 3780.0 & 2.00 & 3787.0 & 3774.0 \\
\hline
\end{tabular}

proximately $9 \%$ of the power at each of these frequency bands was displaced into the adjacent frequencies. Also, there were small variances in these percentages that were not present in the unsmoothed spectra. Finally, the percent power in the $7-\mathrm{Hz}$ frequency band was $2.2 \%$ lower than it was in the $2-$ and $10-\mathrm{Hz}$ frequency bands. Therefore, the nonsmoothed data appear to contain more relevant and stable information than do the smoothed data.

The fourth analysis employed the same methodology that was used in the third analysis. The only difference was that the composite wave in the fourth analysis was composed of 4.5-, 7.5-, and $10.5-\mathrm{Hz}$ waves. The summary statistics for this analysis are contained in Table 4. The percent power spectrum for the unsmoothed composite wave in this analysis had characteristics in common with the unsmoothed percent power spectrum of the $4.5-\mathrm{Hz}$ wave reported in Table 2 . In both instances, there were variances in the percent power in each of the frequency bands, and there was a relatively uneven distribution of power. With the composite wave, we expected that the $4.5-\mathrm{Hz}$ component of the wave would be relatively equally distributed between the $4-$ and $5-\mathrm{Hz}$ frequency bands, that the $7.5-\mathrm{Hz}$ component of the composite wave would be relatively evenly distributed between the 7 - and $8-\mathrm{Hz}$ frequency bands, and so forth. The percent power of the 4.5$\mathrm{Hz}$ component of the complex wave that loads on the 4$\mathrm{Hz}$ frequency band was nearly three times as large as on the $5-\mathrm{Hz}$ frequency band. A similar distribution occurred for the 10.5- $\mathrm{Hz}$ component of the complex wave on the $10-$ and $11-\mathrm{Hz}$ frequency bands. The $7.5-\mathrm{Hz}$ component of the complex wave loaded relatively evenly on the 7and $8-\mathrm{Hz}$ frequency bands. Approximately $8 \%$ of the power in this spectrum was located in frequency bands other than where one would logically expect to find it (in the 3-, 6-, 9-, and $12-\mathrm{Hz}$ frequency bands).

The smoothed complex wave had a more even distribution of the percent power and had less variance than the unsmoothed wave. There was an uneven distribution of the percent power of the three components of the complex wave within the spectrum of the smoothed wave. That is, there was approximately $2.5 \%$ less percent power in the $7-$ and $8-\mathrm{Hz}$ frequency bands combined than there was

Table 4

Percent Power Distribution of a Composite Wave $(4.5,7.5,10.5 \mathrm{~Hz})$

\begin{tabular}{rrrrr}
\hline $\begin{array}{l}\text { Frequency } \\
\text { Band }\end{array}$ & Mean & SD & \multicolumn{2}{c}{ Range } \\
\cline { 4 - 5 } & \multicolumn{5}{c}{ Not Smoothed } & Low \\
\hline 3 & 3.9 & 1.50 & 5.9 & 1.8 \\
4 & 20.8 & 2.90 & 24.6 & 16.6 \\
5 & 7.1 & 1.90 & 9.9 & 4.5 \\
6 & 0.3 & 0.30 & 0.7 & 0.0 \\
7 & 12.5 & 1.80 & 14.7 & 9.9 \\
8 & 12.2 & 2.20 & 15.4 & 9.4 \\
9 & 0.3 & 0.30 & 0.6 & 0.0 \\
10 & 6.7 & 2.20 & 8.5 & 4.5 \\
11 & 20.4 & 2.50 & 24.2 & 17.2 \\
12 & 3.6 & 0.90 & 4.9 & 2.4 \\
& & & &
\end{tabular}

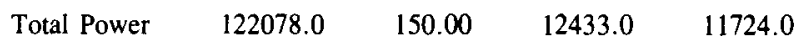

\begin{tabular}{crrrr}
\multicolumn{5}{c}{ Smoothed } \\
3 & 0.2 & 0.03 & 0.2 & 0.1 \\
4 & 17.4 & 0.14 & 17.7 & 17.2 \\
5 & 16.5 & 0.30 & 16.8 & 15.9 \\
6 & .0 & 0.03 & 0.1 & 0.0 \\
7 & 15.8 & 0.20 & 16.4 & 15.6 \\
8 & 15.7 & 0.11 & 15.9 & 15.5 \\
9 & .0 & 0.03 & 0.1 & 0.0 \\
10 & 16.6 & 0.14 & 16.9 & 16.4 \\
11 & 17.2 & 0.20 & 17.4 & 16.8 \\
12 & 0.2 & 0.00 & 0.2 & 0.2 \\
Total Power & 3792.0 & 9.00 & 3813.0 & 3772.0 \\
\hline
\end{tabular}


in either the 4- and $5-\mathrm{Hz}$ or the $10-$ and $11-\mathrm{Hz}$ frequency bands combined. Also, there was less displacement of power from the dominant frequencies to the adjacent frequencies in the smoothed wave than there was in the unsmoothed wave.

\section{CONCLUSIONS}

There are a number of valid conclusions that can be drawn from this study. The first set of conclusions deals with the relationship among the dominant frequency(ies) of the wave, the initial phase angle of the wave, and the frequency bands which are generated by the FFT for data that are not submitted to a smoothing function. If the dominant frequency(ies) of the wave to be analyzed is(are) at or very near an integer value and the frequency bands generated by the FFT are also at or near integer values, then different initial phase angles of the wave analyzed do not greatly affect either the variance of the data or the percent distribution of the power. Conversely, if either the wave analyzed and/or the frequency bands generated by the FFT are not at or near integer values, then the variance of the frequency bands of the power spectra will tend to increase and the distribution of the power in the frequency bands will tend to be less reliable. One possible solution to the problem of matching the frequencies of the waves analyzed and the frequency bands of the power spectrum would be to control it through the use of a different sampling technique. By sampling data so that each sample had the same initial phase angle, the problems associated with the mismatching of the integer values of the frequencies of the wave to be analyzed and the frequency bands of the FFT would be reduced.

The second set of conclusions has to do with using a smoothing function on data prior to the time that they are submitted to a FFT. It is known that the use of a smoothing function on data negates the need to make allowances for differences in the initial phase angles of data. From a positive point of view, the use of a smoothing function tends to reduce the variance of the data. From a negative point of view, the use of a smoothing function tends to reduce the percent power at one or more dominant frequencies while it adds power to adjacent less dominant frequencies, and in situations where there are three dominant frequencies, it tends to reduce the dominance of the frequency located in the center. It appears to us that a smoothing function could be a very powerful tool if used appropriately with EEG data. We do not advise the blind use of this mathematical tool. Instead, we recommend that prior to the analysis of all of the data from a study, a random selection of small samples of the data should be analyzed both with and without a smoothing function. From an initial investigation of the data, decisions can be made as to which of the approaches is most appropriate.

In this study, there were several instances in which a wave with a dominant frequency was analyzed and in the percent power distribution obtained from the FFT the power was distributed across three or more frequency bands. This occurred with both smoothed and unsmoothed waves. If an investigator were to use the power or percent power at only the dominant spectral frequency as the data in a study, he/she would not be using all of the information contained in the original data. In this study, the data displaced into adjacent frequency bands of the spectra represent real information in the study that should be included in the data analyses. This suggests that merging data in frequency bands adjacent to the dominant frequency prior to statistical analysis of data might reduce the variance and increase its reliability and validity. This will be our next area of investigation.

Although we have been conducting EEG research studies for many years, this study has been a profitable experience. Putting aside the laboratory computers, software, and real data that we use on an everyday basis and working for a short time with a computer-generated simulation has given us an opportunity to objectively evaluate our basic data analysis techniques, and there is no doubt that this simulation will influence our future work.

\section{REFERENCES}

Bremner, F. J., Yost, M., \& McKenzie, R. (1982). Computermanaged inferential statistical analysis of EEG data. Behavior Research Methods \& Instrumentation, 14, 300-302.

Bremner, F. J., Yost, M., \& PIKe, A. A. (1984, March). The reliability of the single subject statistics for biofeedback studies. Paper presented at the meeting of the Biofeedback Society of America, Albuquerque, NM.

EDDY, D. R., \& MoISE, S. L. (1985). A comparison of single-subject and traditional statistical designs in steady-state evoked potential research. Behavior Research Methods, Instruments, \& Computers, 17, 323-326.

Jenkins, G. M., \& WatTs, D. G. (1969). Spectral analysis and its application. San Francisco: Hollden-Day.

Perez, F. I., \& Brown, G. A. (1984, March). Single subject statistical design as applied to clinical EMG. Paper presented at the meeting of the Biofeedback Society of America, Albuquerque, NM.

Yost, M., \& BREMNER, F. J. (1985). The use of computer networks in data gathering and data analysis. Behavior Research Methods, Instruments, \& Computers, 17, 157-159.

Yost, M., Cooper, R. A., \& Bremner, F. J. (1983). Fast Fourier analysis: A mathematical and geometric explanation. Behavior Research Methods \& Instrumentation, 15, 258-261. 